\title{
RINGS WHOSE CYCLIC MODULES ARE INJECTIVE OR PROJECTIVE
}

\author{
S. C. GOEL, S. K. JAIN AND SUR JETT SINGH
}

ABSTRACT. The object of this paper is to prove

Theorem. For a ring $R$ the following are equivalent:

(i) Every cyclic right $R$-module is injective or projective.

(ii) $R=S \oplus T$ where $S$ is semisimple artinian and $T$ is a simple

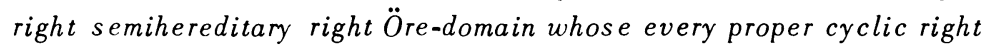
module is injective.

Faith [2] called a ring $R$ a right PCI-ring if each cyclic right $R$-module $C \not R$ is injective, and has shown that a right PCI-ring is either semisimple artinian or else a simple right semihereditary right Öre-domain. The proof of our Theorem provides an alternative and a shorter proof of Faith's result that if $R$ is a regular right PCI-ring (equivalently if $R$ is not a right PCI-domain), then $R$ is semisimple artinian. The existence of PCI-domains which are not division rings is given by Cozzens [1].

Throughout the lemmas the ring $R$ satisfies condition (i) of the Theorem.

Lemma 1. Let $I$ be a two-sided ideal of $R$. Then $R / I$ satisfies (i). Further, if $R / I$ is injective, then $R / I$ is semisimple artinian.

Proof. Clear by Osofsky [3].

Lemma 2. $R$. does not contain an infinite set of central orthogonal idempotents.

Proof. Let $\left\{e_{i}\right\}_{i \in \Lambda}$ be an infinite set of central orthogonal idempotents and $\Lambda^{\prime}$ be an infinite subset of $\Lambda$ such that $\Lambda-\Lambda^{\prime}$ is infinite. Set $A=$ $\bigoplus \Sigma_{i \in \Lambda} e_{i} R, B=\bigoplus \Sigma_{i \in \Lambda-\Lambda^{\prime}} e_{i} R . R / B$ cannot be projective since $B$ is infinitely generated. Hence $R / G$ is semisimple artinian. But $A / B$ is an infinitely generated ideal in $R / B$. This yields a contradiction.

Lemma 3. For any idempotent $e$ in $R$, eR or $(1-e) R$ is a completely reducible injective right $R$-module.

Proof. First we show that the right singular ideal $Z(R)=0$.

If $a \in Z(R)$ then $a E=0$ where $E$ is some essential right ideal of $R$.

Received by the editors July 2, 1974 and, in revised form, September 17, 1974.

AMS (MOS) subject classifications (1970). Primary 16A50, 16A52, 16A64; Secondary 16A12, 16A20.

Key words and phrases. Projective module, injective module, completely reducible module, self-injective ring, (von Neumann) regular ring, prime and semiprime rings, semisimple artinian ring, semihereditary Öre-domain. 
This implies $a R \cong R / E$. If $a R$ is projective then $E=R$ and so $a=0$. If $a R$ is injective then $a R=e R \subset Z(R), e^{2}=e$. This again implies $a R=0$ and thus $a=0$. Now write $R=e R \oplus(1-e) R$. If both $e R$ and $(1-e) R$ are completely reducible then the result is obvious. So suppose $e R$ is not completely reducible. Then there exists a proper essential $R$-submodule $A$ of $e R$. $e R / A$ is then a singular $R$-module and since $Z(R)=(0)$, it cannot be projective. So from $R / A \cong e R / A \oplus(1-e) R$, we get $R / A$ must be injective. Hence $(1-e) R$ is injective. If $(1-e) R$ were also not completely reducible, then, as before, we get $e R$ is injective, and hence $R$ is self-injective. Then Lemma 1 yields that $R$ is semisimple artinian and we are done. Thus in any case if $e R$ is not completely reducible, then $(1-e) R$ is a completely reducible injective module.

Lemma 4. Either $R$ is an integral domain or $R$ has a nonzero socle.

Proof. By Lemma 3 if $R$ has an idempotent $e \neq 0,1$, then $R$ has a nonzero socle. Now suppose $R$ dose not possess idempotents different from 0 and 1. Let $0 \neq a \in R$ and $r(a)=\{x \in R \mid a x=0\}$. Then $a R \cong R / r(a)$. If $a R$ is injective then $a R=(0)$ or $a R=R$. The former implies $a=0$ and the latter implies $R$ is right self-injective and hence semisimple artinian, consequently a division ring. If $a R$ is projective then $r(a)=(0)$ or $r(a)=R$. The latter is not possible. Hence $r(a)=0$ and $R$ is an integral domain.

Lemma 5. If $R$ has no nontrivial central idempotents then either $R$ is simple artinian or $R$ is a simple right semibereditary right Öre-domain.

Proof. If $R$ is a domain then $R$ is a right PCI-ring and hence, by Faith [2, Propositions 5,17], $R$ is a simple right semihereditary right Öre-domain. If $R$ is not a domain then $R$ has a nonzero socle $S$. From Lemma 3 every minimal right ideal of the form $e R, e=e^{2} \in R$, is injective. Thus hypothesis (i) yields that every minimal right ideal $a R$ of $R$ is generated by an idempotent. But then it follows immediately that $R$ is semiprime. Indeed $R$ can be shown to be prime since $R$ has no nontrivial central idempotents. In case $R / S$ is projective, then $S=e R$ where $e$ is a central idempotent, so that $R=S$ is simple artinian. If $R / S$ is injective then $R / S$ is semisimple artinian, and hence $R$ is regular. Thus $R$ is a primitive regular ring with nonzero socle. Let $\hat{R}$ denote the maximal right quotient ring of $R$. Since every minimal right ideal is injective, the socle of $\hat{R}=$ socle of $R=S$. If $S$ is finitely generated then $S=R=\hat{R}$ and hence $R$ is simple artinian. So assume that $S$ is not finitely generated. Then there exists right ideals $K_{1}, K_{2}$ in $S$ such that $S=K_{1} \oplus K_{2}$ and $S \approx K_{1} \approx K_{2}$. Since $K_{1}$ is infinitely generated, $R / K_{1}$ is injective. Also $R / K_{1}$ contains $\left(K_{1} \oplus K_{2}\right) / K_{1} \approx K_{2} \approx S$. Hence $\hat{R}_{R}=\hat{S}_{R}$ is embeddable in $R / K_{1}$. This implies $\hat{R}=x R$ for some $x \in \hat{R}$, so there exists $a \in R$ such that $x a=1$. This implies $a+S$ is invertible in the semisimple 
artinian ring $R / S$. Thus there exists $y+S$ in $R / S$ such that ay $-1 \in S$. This yields that $x \in R$. Hence $R=\hat{R}$ which by Lemma 1 implies $R$ is simple artinian. This proves the lemma.

Proof of the Theorem. Assume (i). By Lemma 2 we can write $R=R_{1} \oplus$ $R_{2} \oplus \ldots \oplus R_{n}$ where $R_{i}$ are rings having no nontrivial central idempotents. Then by Lemma 3 we get all $R_{i}$, excepting at most one, are semisimple artinian. Hence either $R$ is semisimple artinian or $R=S \boxplus T$ where $S$ is semisimple artinian and $T$ has no nontrivial central idempotents. Since $T$ also satisfies (i), Lemma 5 completes the proof. The converse is clear.

\section{REFERENCES}

1. J. H. Cozzens, Homological properties of the ring of differential polynomials, Bull. Amer. Math. Soc. 76 (1970), 75-79. MR 41 \#3531.

2. C. Faith, When are proper cyclics injective? Pacific J. Math. 45 (1973), 97112. MR $47 \# 8610$.

3. B. L. Osofsky, Noninjective cyclic modules, Proc. Amer. Math. Soc. 19 (1968), 1383-1384. MR $38 \# 185$.

DEPARTMENT OF MATHEMATICS, OHIO UNIVERSITY, ATHENS, OHIO 45701 (Current address of S. C. Goel and S. K. Jain)

Current address (Surjeet Singh): Department of Mathematics, Guru Nanak University, Amritsar (Panjab), India 